\title{
Qualitative Properties of Local Random Invariant Manifolds for SPDEs with Quadratic Nonlinearity
}

\author{
Dirk Blömker \\ Institut für Mathematik \\ Universität Augsburg \\ 86135 Augsburg \\ dirk.bloemker@math.uni-augsburg.de \\ Wei Wang \\ School of Mathematics \\ University of Adelaide \\ 5005 Adelaide Australia \\ w.wang@adelaide.eu.au \\ \& \\ Department of Mathematics \\ Nanjing University \\ 210093 Nanjing China \\ wangweinju@yahoo.com.cn
}

April 4, 2022

\begin{abstract}
The qualitative properties of local random invariant manifolds for stochastic partial differential equations with quadratic nonlinearities and multiplicative noise is studied by a cut off technique. By a detail estimates on the Perron fixed point equation describing the local random invariant manifold, the structure near a bifurcation is given.
\end{abstract}




\section{Introduction}

Stochastic partial differential equations SPDEs arise as macroscopic mathematical models of complex systems under random influences. There have been rapid progresses in this area [18, 21, 14, 25, 4, 19. More recently, SPDEs have been investigated in the context of random dynamical systems (RDS) [1]; see [8, 10, 11, 12, 13, 22, 16, 17, e.g.], among others.

Invariant manifolds are special invariant sets locally represented by graphs in state spaces (function spaces) where the solution process of the system is defined. A random invariant manifold provides a geometric structure to reduce stochastic dynamics. In fact the dynamics of the system is completely determined by that on the globally attracting invariant manifold. Nevertheless, due to the inherit non-autonomous nature of the SPDE, these manifolds move in time. So one can reduce the system to a lower dimensional system on the invariant manifold together with the dynamics of that manifold.

There are some results on the existence of random invariant manifold for a class of stochastic partial differential equations with Lipschitz nonlinearity, [16, 17, 20] etc. In [8] an estimate of dimension of the local invariant manifold is given, in order to study a pitchfork type bifurcation for stochastic reactiondiffusion equations with cubic nonlinearity.

In the recent work [24], by a detail study of the properties of flow, an invariant manifold reduction of a class stochastic partial differential equations with Lipschitz nonlinearity is obtained. The reduced equation is a stochastic differential equation defined on a finite dimensional invariant manifold. Moreover in [15] the expansion in leading order terms of a random invariant manifold for noise-strength to 0 is considered.

There is a difficulty in reducing infinite dimensional stochastic systems with non-Lipschitz nonlinearity to a lower dimensional system on an invariant manifold. But it can be done near an equilibrium solution by introducing a cut-off function near the equilibrium solution such that the nonlinearity becomes globally Lipschitz in an approximate space. Similar ideas are already used in [24] and [8]. In this situation for the equation with cut-off the global approach in 24] could be followed, and the result for the original equation is then a local one.

However, let us point out that the invariant manifold is still moving in the infinite dimensional space. It is in general difficult to see clearly the dynamics of the reduced system even though it is essentially finite dimensional. Therefore we aim at the local shape of the invariant manifold.

At the same time an amplitude equation is an important tool to describe qualitatively the dynamics of stochastic systems near a change stability, which has been studied heavily for additive noise $([2,3$, , 5, e.g.] $])$ and for 
multiplicative noise (see [4, 6]). In contrast to random invariant manifolds, which move in time, the dynamics studied via amplitude equations approximates the dynamics on a given fixed vector space, and the essential dynamics is given by a stochastic ordinary differential equation on dominant modes. The drawback in this case is that the results hold only with high probability. Results that hold almost surely are not possible to obtain.

In this paper we study the properties of the random invariant manifold locally. In fact we consider properties of local invariant manifolds for equations near a change of stability with quadratic nonlinearities and multiplicative noise. One of the simplest examples is a Burgers-type equation

$$
\partial_{t} u=\partial_{x}^{2} u+u+\nu u+\frac{1}{2} \partial_{x} u^{2}+\sigma u \circ \dot{W}
$$

subject to Dirichlet boundary conditions on $[0, \pi]$ with one-dimensional multiplicative noise of Stratonovic-type.

There are many examples of SPDEs near its first change of stability, where our results apply. For instance, we can consider the Kuramoto-Sivashinsky equation, a model from surface growth (cf. [7]), Navier-Stokes equations with an additional linear term, or the Rayleigh-Benard system near the convective instability.

For our main results we reduce the cut-off equation of (1) to a stochastic differential equation on an attracting random invariant manifold in a small cut-off ball. Our results extend [9], where the local dimension of a random invariant manifold near a fixed point is studied. Using the linearization in our case the local dimension is one, but here we show that with high probability the manifold is locally the graph of a quadratic function over a one-dimensional space (cf. Theorem 3).

Let us compare our results with amplitude equations. Typically, one assumes there the scaling $\sigma=\varepsilon, \nu=\nu_{0} \varepsilon^{2}$ for some small $\varepsilon$ with $\left|\nu_{0}\right| \leq 1$. In that scaling our Burgers-type equation reads

$$
\partial_{t} u=\partial_{x}^{2} u+u+\nu_{0} \varepsilon^{2} u+\frac{1}{2} \partial_{x} u^{2}+\varepsilon u \circ \dot{W} .
$$

In [4] it was shown that for solutions of size $\mathcal{O}(\varepsilon)$ with probability higher than $1-C_{p} \varepsilon^{p}$ for all $p>1$

$$
u(t, x) \approx \varepsilon a\left(\varepsilon^{2} t\right) \sin
$$

on intervals of length $\mathcal{O}\left(\varepsilon^{-2}\right)$, where

$$
\partial_{T} a=\nu_{0} a-\frac{1}{12} a^{3}+a \circ \dot{W}
$$

Our main results of this paper show for $\nu$ and $\sigma$ small, but without scaling assumption, that there is a time dependent random Lipshitz map $\phi(t, \omega, \cdot)$ : 
$\mathbb{R} \rightarrow \mathbb{R}$ describing locally the random invariant manifold such that near 0 the flow along the manifold is

$$
\partial_{t} a=\nu a+a \phi(t, a)+\sigma a \circ \dot{W} .
$$

Moreover, for a given $t$ the probability that

$$
\phi(t, a) \approx-\frac{1}{12} a^{2}
$$

is larger than $1-C \exp \{-1 / \varepsilon\}$.

The rest of the paper is organized as follows. In Section 2, assumptions and main results are presented. Section 3 states the results on existence of random invariant manifolds using a cut-off technique. While Section 4 provides results on the local structure of the manifold.

\section{Setting and Results}

Consider the following abstract equation

$$
\partial_{t} u=-L u+\nu u+B(u, u)+\sigma u \circ \dot{W}, \quad u(0)=u_{0} \in H
$$

where $W$ is a standard real valued Brownian motion, and the noise is in the Stratonovic sense. The real constants $\nu$ and $\sigma$ describe the distance from the bifurcation and the noise strength, respectively. Assume $H$ is a real separable Hilbert space with norm $\|\cdot\|$ and scalar product $\langle\cdot, \cdot\rangle$.

We make the following assumptions.

Assumption $\mathbf{A}_{1}$ Let $L$ be a non-negative self-adjoint unbounded operator with compact resolvent in the Hilbert space $H$, and suppose that $\left\{e_{k}\right\}_{k=1}^{\infty}$ and $\left\{\lambda_{k}\right\}_{k=1}^{\infty}$ is an orthonormal basis of eigenfunctions and the corresponding ordered eigenvalues $\left(\lambda_{k+1} \geq \lambda_{k}\right)$.

Assumption $\mathbf{A}_{2}$ The kernel of $L, H_{c}:=\operatorname{span}\left\{e_{1}, \ldots, e_{N}\right\}$, is finite dimensional, i.e. $\lambda_{1}=\ldots=\lambda_{N}=0$ and $\lambda_{*}=\lambda_{N+1}>0$.

Denote by $P_{c}$ the orthogonal projection from $H$ to $H_{c}$. Furthermore, $P_{s}=$ $I-P_{c}$ and let $L_{s}=P_{s} L$. In the following we use the subscript 'c' always for projection onto $H_{c}$ and 's' for projection onto $H_{s}$.

Remark 1. Note that the assumption that the eigenfunctions form an orthonormal set is for simplicity. We could relax this, assuming that $P_{c}$ is a continuous projection commuting with $L$, which is no longer self-adjoint in that case. 
Let us now introduce the interpolation spaces $H^{\alpha}, \alpha>0$, as the domain of $L^{\alpha / 2}$ endowed with scalar product $\langle u, v\rangle_{\alpha}=\left\langle u,(1+L)^{\alpha} v\right\rangle$ and corresponding norm $\|\cdot\|_{\alpha}$. Furthermore, we identify $H^{-\alpha}$ as the dual of $H^{\alpha}$ with respect to the inner product in $H$.

We make the following assumption on the nonlinearity.

\section{Assumption $\mathbf{A}_{3}$}

For some $\alpha \in(0,1)$, let $B: H \times H \rightarrow H^{-\alpha}$ be a bounded bilinear and symmetric operator, i.e. $B(u, \bar{u})=B(\bar{u}, u)$ and there is a constant $C_{B}>0$ such that $\|B(u, \bar{u})\|_{-\alpha} \leq C_{B}\|u\|\|\bar{u}\|$. Also denote by $\langle\cdot, \cdot\rangle$ the dual paring between $H^{-\alpha}$ and $H^{\alpha}$. Then suppose that for $B(u) \triangleq B(u, u)$

$$
\langle B(u), u\rangle=0 \quad \text { for all } u \in H^{\alpha} .
$$

In the following we denote $P_{c} B(\cdot, \cdot)$ by $B_{c}(\cdot, \cdot)$ and $P_{s} B(\cdot, \cdot)$ by $B_{s}(\cdot, \cdot)$. From bilinearity and boundedness, we immediately obtain a local Lipschitz condition for $B$. To be more precise, for all $R>0$

$$
\|B(u)-B(\bar{u})\|_{-\alpha}=\|B(u-\bar{u}, u+\bar{u})\|_{-\alpha} \leq 2 R C_{B}\|u-\bar{u}\|
$$

for all $u, \bar{u} \in H$ with $\|u\| \leq R$ and $\|\bar{u}\| \leq R$.

Let us remark that the symmetry of $B$ is not necessary, but it simplifies for instance expansions of $B(u+v)$. Moreover, one can without loss of generality always assume that the quadratic form is given by a symmetric $B$. If not one can consider $\tilde{B}(u, v)=\frac{1}{2}(B(u, v)+B(v, u))$.

\section{Random Dynamical Systems}

Before giving our main result, we recall some basic theory of random dynamical systems. We will work on the canonical probability space $\left(\Omega_{0}, \mathcal{F}_{0}, \mathbb{P}\right)$ where the sample space $\Omega_{0}$ consists of the sample paths of $W(t)$. To be more precise $W$ is the identity on $\Omega_{0}$, with

$$
\Omega_{0}=\{w \in C([0, \infty), \mathbb{R}): w(0)=0\}
$$

and $\mathbb{P}$ the Wiener measure. For more details see [1].

Let $\theta_{t}:\left(\Omega_{0}, \mathcal{F}_{0}, \mathbb{P}\right) \rightarrow\left(\Omega_{0}, \mathcal{F}_{0}, \mathbb{P}\right)$ be a metric dynamical system (driven system), that is,

- $\theta_{0}=i d$,

- $\theta_{t} \theta_{s}=\theta_{t+s}$, for all $s, t \in \mathbb{R}$,

- the map $(t, \omega) \mapsto \theta_{t} \omega$ is measurable and $\theta_{t} \mathbb{P}=\mathbb{P}$ for all $t \in \mathbb{R}$. 
On $\Omega_{0}$ the map $\theta_{t}$ is the shift

$$
\theta_{t} \omega(\cdot)=\omega(\cdot+t)-\omega(t), \quad t \in \mathbb{R}, \omega \in \Omega_{0} .
$$

Definition 1. Let $(\mathcal{X}, d)$ be a metric space with Borel $\sigma$-algebra $\mathcal{B}$, then a random dynamical system on $(\mathcal{X}, d)$ over $\theta_{t}$ on $\left(\Omega_{0}, \mathcal{F}_{0}, \mathbb{P}\right)$ is a measurable map

$$
\begin{aligned}
\varphi: \mathbb{R}^{+} \times \Omega_{0} \times \mathcal{X} & \rightarrow \mathcal{X} \\
(t, \omega, x) & \mapsto \varphi(t, \omega) x
\end{aligned}
$$

having the following cocycle property

$$
\varphi(0, \omega) x=x, \quad \varphi\left(t, \theta_{\tau} \omega\right) \circ \varphi(\tau, \omega) x=\varphi(t+\tau, \omega) x
$$

for $t, \tau \in \mathbb{R}^{+}, x \in \mathcal{X}$ and $\omega \in \Omega$.

A $\operatorname{RDS} \varphi$ is continuous or differentiable if $\varphi(t, \omega): \mathcal{X} \rightarrow \mathcal{X}$ is continuous or differentiable (see [1] for more details on RDS).

It is well known that in order to show that (44) generates an RDS, one can rely on a random transformation to a random PDE. For this we introduce the following real-valued stationary process.

Definition 2. Define

$$
z(t)=z\left(\theta_{t} \omega\right)
$$

on $\Omega_{0}$, where

$$
z(\omega)=\sigma \int_{-\infty}^{0} e^{s} \omega(s) d s
$$

Now the mapping $t \mapsto z\left(\theta_{t} \omega\right)$ is continuous and solves

$$
d z+z d t=\sigma d \omega \quad \text { or } \quad z\left(\theta_{t} \omega\right)=z(\omega)-\int_{0}^{t} z\left(\theta_{s} \omega\right) d s+\sigma \omega(t) .
$$

Moreover,

$$
\lim _{t \rightarrow \pm \infty} \frac{\left|z\left(\theta_{t} \omega\right)\right|}{|t|}=0 \text { and } \lim _{t \rightarrow \pm \infty} \frac{1}{t} \int_{0}^{t} z\left(\theta_{\tau} \omega\right) d \tau=0 \text { for a.e. } \omega \in \Omega_{0}
$$

The above properties hold in a $\theta_{t}$ invariant set $\Omega \subset \Omega_{0}$ of full probability, see [16]. In the following, we frequently use the shorthand notation $z(t)=z\left(\theta_{t} \omega\right)$.

By the transformation

$$
v(t)=e^{-z(t)} u(t)
$$


Equation (4) becomes

$$
\partial_{t} v=-L v+z v+\nu v+e^{z} B(v, v),
$$

which is an evolutionary equation with random stationary coefficient. Then for almost all $\omega \in \Omega$, by the same discussion for the wellposedness to deterministic evolutionary equation [23], for any $t_{0}<T \in \mathbb{R}$ and $v_{0} \in H$ there is a unique solution $v\left(t, \omega ; t_{0}, v_{0}\right) \in C\left(t_{0}, T ; H\right)$ of equation (111) with $v\left(t_{0}\right)=v_{0}$ and the map $v_{0} \mapsto v\left(t, \omega ; t_{0}, v_{0}\right)$ is continuous for all $t \geq t_{0}$. Then by the stationary transformation (10) we have the following result.

Theorem 1. Under Assumptions $A_{1}-A_{3}$, (4) generates a continuous $R D S$ $\varphi(t, \omega)$ on $H$.

For a continuous random dynamical system $\varphi$ on $\mathcal{X}$ given by Definition 1, we need the following notions to describe its dynamical behavior. First for any subset $M \subset \mathcal{X}$ define $d(M)=\sup _{x \in M} d(x, 0)$ with $0 \in \mathcal{X}$ is the zero element in $\mathcal{X}$ and define $B_{R}(0)=\{x \in \mathcal{X}: d(x, 0)<R\}$.

Definition 3. A collection $M=M(\omega)_{\omega \in \Omega}$ of non-empty closed sets $M(\omega) \subset$ $\mathcal{X}, \omega \in \Omega$ is a random set, if

$$
\omega \mapsto \inf _{y \in M(\omega)} d(x, y)
$$

is a real valued random variable for any $x \in \mathcal{X}$.

Definition 4. A random set $M(\omega)$ is called a tempered absorbing set for a random dynamical system $\varphi$ if for any bounded set $K \subset \mathcal{X}$ there exists $t_{K}(\omega)$ such that $\forall t \geq t_{K}(\omega)$

$$
\varphi(t, \omega) K \subset M\left(\theta_{t} \omega\right) .
$$

and for all $\varepsilon>0$

$$
\lim _{t \rightarrow \infty} e^{-\varepsilon t} d\left(M\left(\theta_{t} \omega\right)\right)=0, \text { for a.e. } \omega \in \Omega .
$$

For more details about random set we refer to [12].

Definition 5. A random set $M(\omega)$ is called a positive invariant set for a random dynamical system $\varphi(t, \omega)$ if

$$
\varphi(t, \omega) M(\omega) \subset M\left(\theta_{t} \omega\right), \text { for } t \geq 0 .
$$

If $M(\omega)=\left\{x_{1}+\psi\left(\omega, x_{1}\right) \mid x_{1} \in \mathcal{X}_{1}\right\}$ is the graph of a random Lipschitz mapping

$$
\psi(\omega, \cdot): \mathcal{X}_{1} \rightarrow \mathcal{X}_{2}
$$

with $\mathcal{X}=\mathcal{X}_{1} \oplus \mathcal{X}_{2}$, then $M(\omega)$ is called a Lipschitz invariant manifold of $\varphi$. 
For further details about the random invariant manifold theory, see [16].

Definition 6. System (4) is said to have a local random invariant manifold (LRIM) with radius $R$, if there is a random set $\mathcal{M}^{R}(\omega)$, which is defined by the graph of a random continuous function $\psi(\omega, \cdot): \overline{B_{R}(0)} \cap \mathcal{X}_{1} \rightarrow \mathcal{X}_{2}$, such that for all bounded sets $B$ in $B_{R}(0) \subset \mathcal{X}_{1}$ we have

$$
\varphi(t, \omega)\left[\mathcal{M}^{R}(\omega) \cap B\right] \subset \mathcal{M}^{R}\left(\theta_{t} \omega\right)
$$

for all $t \in\left(0, \tau_{0}(\omega)\right)$ with

$$
\tau_{0}(\omega)=\tau_{0}(\omega, B)=\inf \left\{t \geq 0: \varphi(t, \omega)\left[\mathcal{M}^{R}(\omega) \cap B\right] \not \subset B_{R}(0)\right\} .
$$

In [20] the existence of a random invariant set on a tempered ball around 0 was established. This should be possible, if we have a LRIM. But we will not focus on that. In the following we are aiming on local properties of the LRIM.

Due to the method of proof, where we rely on a cut-off at radius $R$, we always obtain a globally defined Lipschitz invariant manifold $\mathcal{M}(\omega)$ for the system with cut-off. Now, as both flows agree on $B_{R}(0)$ it is easy to check that $\mathcal{M}^{R}(\omega)=\mathcal{M}(\omega) \cap B_{R}(0)$ defines a LRIM. In the following, we will mainly work with the globally defined $\mathcal{M}(\omega)$.

\section{Main Results}

We prove the following theorem in Section 3 , See Theorems 4 and 5 , This is based on the properties for the system with cut-off.

Theorem 2. (Existence) Under Assumptions $A_{1}-A_{3}$, the random $d y$ namical system $\varphi(t, \omega)$ defined by (4) has a $\operatorname{LRIM} \mathcal{M}^{R}(\omega)$ for sufficiently small $R>0$. This manifold is given as the graph of a random Lipschitz map $h(\omega, \cdot): H_{c} \rightarrow H_{s}:$

$$
\mathcal{M}^{R}(\omega)=\left\{\left(\xi, e^{z(\omega)} h\left(\omega, e^{-z(\omega)} \xi\right)\right): \xi \in H_{c}\right\} .
$$

Moreover, if $\lambda_{*}>4 \nu$, then the $\operatorname{LRIM} \mathcal{M}^{R}(\omega)$ is locally exponentially attracting almost surely in the small ball $B_{R}(0)$. That is for any $\left\|u_{0}\right\|<R$

$$
\operatorname{dist}\left(\varphi(t, \omega) u_{0}, \mathcal{M}^{R}\left(\theta_{t} \omega\right)\right) \leq 2 R D(t, \omega) e^{-\lambda_{*} t}
$$

for all $t<\tau_{0}(\omega)=\inf \left\{t>0: \varphi(t, \omega) u_{0} \notin B_{r}(0)\right\}$ with $D(t, \omega)$ is a tempered increasing process, see (29). 
We are interested in the property of solutions on the LRIM $\mathcal{M}^{R}(\omega)$ and the dynamics of $\mathcal{M}^{R}(\omega)$ itself near the first bifurcation $\nu=0$.

It is proved that

Theorem 3. (Local Shape) Let Assumptions $A_{1}-A_{3}$ be true. Suppose $\sigma>0,|\nu|<\sigma$ and $R \leq 1$, and let $h$ be the fixed point given by Theorem 4. Then for $\sigma \rightarrow 0$,

$$
\left\|e^{z(\omega)} h\left(\omega, e^{-z(\omega)} \xi\right)-L_{s}^{-1} B_{s}(\xi, \xi)\right\| \leq C\left(\|\xi\|+R^{2}+\sqrt{\sigma}\right) \cdot\|\xi\|^{2},
$$

holds with probability larger than $1-C \exp \{-1 / \sqrt{\sigma}\}$ for all $\|\xi\| \leq \frac{1}{2} R$.

Remark 2. The previous result will not apply to deterministic equations, as we always assume $|\nu|<\sigma$, but it allows for large radii $R<1$ for the cut-off.

Let us give a more general example than the Burgers' equation already mentioned. If we consider the space $H_{c}$ being one-dimensional, then we can write $\xi=\alpha \cdot e$ for a basis function $e \in H_{c}$.

Thus the invariant manifold $\mathcal{M}^{R}(\omega)$ is locally given (with high probability as the graph of

$$
L_{s}^{-1} B_{s}(\xi, \xi)=\alpha^{2} L_{s}^{-1} B_{s}(e, e)=: \alpha^{2} v_{s} \in H_{s} .
$$

Now $\mathcal{M}^{R}(\omega)$ lies approximately in the plane spanned by $e$ and $v_{s}$ and is given by a parabola, unless $v_{s}=0$. In that case the manifold is approximately flat.

Let us finally comment on the flow on the manifold. Using Theorem 2 , it is easy to describe the flow along the invariant manifold $\mathcal{M}(\omega)$ on a small ball of radius $R / 2$ around 0 by the following equation

$$
\partial_{t} u_{c}=\nu u_{c}+P_{c} B^{(R)}\left(u_{c}+e^{z\left(\theta_{t} \omega\right)} h\left(\theta_{t} \omega, e^{-z\left(\theta_{t} \omega\right)} u_{c}\right)\right)+\sigma u_{c} \circ \dot{W}(t) .
$$

By Theorem 3 we can replace $e^{z\left(\theta_{t} \omega\right)} h\left(\theta_{t} \omega, e^{-z\left(\theta_{t} \omega\right)} u_{c}\right)$ by

$$
L_{s}^{-1} B_{s}\left(u_{c}, u_{c}\right)+r(t),
$$

where the probability of $r(t)$ being large is bounded by $C \exp \{-1 / \sqrt{\sigma}\}$.

This finally yields an equation, where

$$
\left.\partial_{t} u_{c}=\nu u_{c}+P_{c} B^{(R)}\left(u_{c}+L_{s}^{-1} B_{s}\left(u_{c}, u_{c}\right)\right)\right)+\sigma u_{c} \circ \dot{W}(t) .
$$

This rederives an amplitude equation for the equation. Nevertheless, for a detailed analysis of the flow on $\mathcal{M}$, we would need bound on $r$, which are uniform in time. This will be postponed to future work.

To conclude the presentation of the main results, we remark that it should be straightforward to generalize the presented results to higher, but finite dimensional noise. 


\section{Existence of Local Invariant Manifold}

In this section we construct a LRIM for system (4) for small $R>0$ and prove its exponentially attracting property. For this we rely on a cut-off technique given by the following definition. As explained after Definition 6 (cf. also [8, 20]), the LRIM is given by the RIM for the cut-off system.

Definition 7. (cut-off) Let $\chi: H \rightarrow \mathbb{R}$ be a bounded smooth function such that $\chi(u)=1$ if $\|u\| \leq 1$ and $\chi(u)=0$ if $\|u\| \geq 2$. For any $R>0$, we define $\chi_{R}(u)=\chi(u / R)$ for all $u \in H$.

Given a radius $R>0$ we define

$$
B^{(R)}(u)=\chi_{R}(u) B(u, u) .
$$

Now by Assumption $\left(\mathrm{A}_{3}\right)$ for given $1>\alpha>0$, the operator $B^{(R)}$ is globally Lipschitz-continuous from space $H$ to $H^{-\alpha}$ with Lipschitz constant

$$
\operatorname{Lip}_{H, H^{-\alpha}}\left(B^{(R)}\right)=L_{R}:=2 R C_{B} .
$$

Consider now the following cut-off system

$$
\partial_{t} u=-L u+\nu u+B^{(R)}(u)+\sigma u \circ \dot{W}, \quad u(0)=u_{0} .
$$

As before by the transformation $v=u e^{-z}$ (with $z=z(t)=z\left(\theta_{t} \omega\right)$ ), we have

$$
v_{t}=-L v+z v+\nu v+e^{-z} B^{(R)}\left(e^{z} v\right), \quad v(0)=u_{0} e^{-z(0)} .
$$

In order to obtain a random invariant manifold for random dynamical system $\varphi^{R}(t, \omega)$ defined by the above stochastic equation (15) we study the transformed equation (16). By the projections $P_{c}$ and $P_{s}$ equation (16) is split into

$$
\begin{array}{rlrl}
\partial_{t} v_{c}= & \nu v_{c}+z v_{c}+P_{c} e^{-z} B^{(R)}\left(e^{z} v\right), & v_{c}(0)=P_{c} u_{0} e^{-z(0)}, \\
\partial_{t} v_{s}=-L_{s} v_{s}+\nu v_{s}+z v_{s}+P_{s} e^{-z} B^{(R)}\left(e^{z} v\right), & v_{s}(0)=P_{s} u_{0} e^{-z(0)}
\end{array} .
$$

We use the Lyapunov-Perron method on the following random space with random norm depending on $\omega \in \Omega$. The process $z$ was defined in (9).

Definition 8. For $-\nu<\eta<\lambda_{*}-\nu$ define the Banach space

$$
C_{\eta}^{-}=\left\{v \in C((-\infty, 0], H): \sup _{t \leq 0}\left\{e^{\eta t-\int_{0}^{t} z(s) d s}\|v(t)\|\right\}<\infty\right\}
$$

with norm

$$
\|v\|_{C_{\eta}^{-}}=\sup _{t \leq 0}\left\{e^{\eta t-\int_{0}^{t} z(\tau) d \tau}\|v(t)\|\right\}<\infty
$$


Definition 9. Define the nonlinear operator $\mathcal{T}$ on $C_{\eta}^{-}$for given $\xi \in H_{c}$ and $\omega \in \Omega_{0}$ as

$$
\begin{aligned}
\mathcal{T}(v, \xi)(t)= & e^{\nu t+\int_{0}^{t} z(s) d s} \xi+\int_{0}^{t} e^{\nu(t-\tau)+\int_{\tau}^{t} z(r) d r} e^{-z(\tau)} P_{c} B^{(R)}\left(v(\tau) e^{z(\tau)}\right) d \tau \\
& +\int_{-\infty}^{t} e^{\left(-L_{s}+\nu\right)(t-\tau)+\int_{\tau}^{t} z(r) d r} e^{-z(\tau)} P_{s} B^{(R)}\left(\left(v(\tau) e^{z(\tau)}\right) d \tau .(18)\right.
\end{aligned}
$$

By the Lipschitz property of $B^{(R)}$ (cf. (14)) it can be verified directly that for any $\xi \in H_{c}$ and $\omega \in \Omega_{0}, \mathcal{T}(\cdot, \xi): C_{\eta}^{-} \rightarrow C_{\eta}^{-}$. Further, a short calculation shows that it is a Lipschitz continuous map. To be more precise, for any $v$, $\bar{v} \in C_{\eta}^{-}$

$$
\begin{aligned}
& {[\mathcal{T}(v)(t)-\mathcal{T}(\bar{v})(t)] e^{-\int_{0}^{t} z(r) d r}} \\
& =\quad+\int_{0}^{t} e^{\nu(t-\tau)+\int_{0}^{\tau} z(r) d r-z(\tau)}\left[P_{c} B^{(R)}\left(v(\tau) e^{z(\tau)}\right)-P_{c} B^{(R)}\left(\bar{v}(\tau) e^{z(\tau)}\right)\right] d \tau \\
& \quad+\int_{-\infty}^{t} e^{\left(-L_{s}+\nu\right)(t-\tau)-\int_{0}^{\tau} z(r) d r-z(\tau)}\left[P _ { s } B ^ { ( R ) } \left(\left(v(\tau) e^{z(\tau)}\right)-P_{s} B^{(R)}\left(\left(\bar{v}(\tau) e^{z(\tau)}\right)\right] d \tau .\right.\right.
\end{aligned}
$$

Now we use that for all $t>0$ and all $u \in H_{s}$

$$
\left\|e^{L t} u\right\| \leq M_{\alpha, \lambda} e^{-t \lambda} t^{-\alpha}\|u\|_{-\alpha}
$$

for some $\lambda<\lambda_{*}$ sufficiently close, together with (14) and the estimate $\left\|P_{c} v\right\| \leq C_{\alpha}\left\|P_{c}\right\|_{-\alpha}$ for some constant $C_{\alpha}$.

We obtain for $0<\eta+\nu<\lambda$

$$
\begin{aligned}
\|\mathcal{T}(v)-\mathcal{T}(\bar{v})\|_{C_{\eta}^{-}} \leq & \int_{0}^{t} e^{(\nu+\eta)(t-\tau)} C_{\alpha} L_{R}\|v-\bar{v}\|_{C_{\eta}^{-}} d \tau \\
& +\int_{-\infty}^{t} e^{(-\lambda+\nu+\eta)(t-\tau)} M_{\alpha, \lambda} L_{R}\|v-\bar{v}\|_{C_{\eta}^{-}} d \tau \\
\leq & L_{R}\left[\frac{C_{\alpha}}{\eta+\nu}+M_{\alpha, \lambda} \frac{\Gamma(1-\alpha)}{(\lambda-\eta-\nu)^{1-\alpha}}\right]\|v-\bar{v}\|_{C_{\eta}^{-}} .
\end{aligned}
$$

Note that our bound on $\operatorname{Lip}(\mathcal{T})$ is actually independent of $\xi$ and $\omega$.

Now for all $\xi \in H_{c}$ and $\omega \in \Omega_{0}$ the operator $\mathcal{T}: C_{\eta}^{-} \rightarrow C_{\eta}^{-}$is a contraction provided $R$ is sufficiently small such that

$$
L_{R}\left[\frac{C_{\alpha}}{\eta+\nu}+M_{\alpha, \lambda} \frac{\Gamma(1-\alpha)}{(\lambda-\eta-\nu)^{1-\alpha}}\right]<1 .
$$

The celebrated theorem of Banach yields the following theorem. 
Theorem 4. Suppose $0<\eta+\nu<\lambda_{*}$ and (20). Then the operator $\mathcal{T}$ has a unique fixed point $v^{*}=v^{*}(\omega, \xi) \in C_{\eta}^{-}$.

Define $h(\omega, \xi)=P_{s} v^{*}(0, \omega ; \xi)$. Thus, by Definition 9

$$
h(\omega, \xi)=\int_{-\infty}^{0} e^{\left(L_{s}-\nu\right) \tau+\int_{\tau}^{0} z(r) d r} e^{-z(\tau)} P_{s} B^{(R)}\left(v^{*}(\tau, \xi) e^{z(\tau)}\right) d \tau .
$$

Then by the same discussion as in [17],

$$
\mathcal{M}_{\text {cut }}^{R}(\omega)=\left\{\left(\xi, e^{z(\omega)} h\left(\omega, e^{-z(\omega)} \xi\right)\right): \xi \in H_{c}\right\}
$$

is a random invariant manifold for the random dynamical system $\varphi^{R}(t, \omega)$, which is the graph of $e^{z(\omega)} h\left(\omega, e^{-z(\omega)} \xi\right)$. Now define a Lipschitz mapping $\psi$ by

$$
\begin{aligned}
\psi(\omega, \cdot): H_{c} \cap B_{R}(0) & \rightarrow H_{s}, \\
\xi & \rightarrow \psi(\omega, \xi)=e^{z(\omega)} h\left(\omega, e^{-z(\omega)} \xi\right) .
\end{aligned}
$$

Then, as already indicated after the definition, it is easy to check, that

$$
\mathcal{M}^{R}(\omega)=\operatorname{graph}(\psi(\omega, \cdot))=\mathcal{M}_{c u t}^{R}(\omega) \cap B_{R}(0)
$$

defines a LRIM of the random dynamical system $\varphi(t, \omega)$.

Now we prove the attracting property of the random invariant manifold $\mathcal{M}_{\text {cut }}^{R}(\omega)$ for equation (15). We follow the approach of [24]. It is enough to prove the cone invariance property:

Lemma 1. Fix $\delta>0$ and define the cone

$$
\mathcal{K}_{\delta}=\left\{u \in H:\left\|u_{s}\right\|<\delta\left\|u_{c}\right\| \|\right\} .
$$

Suppose that $R$ is sufficiently small such that

$$
\lambda_{*} \geq 2\left(1+\frac{1}{\delta}\right)^{2} L_{R}^{2}+4(1+\delta) L_{R}
$$

and

$$
\lambda_{*}>4 \nu+2 L_{R}^{2}\left(1+\frac{1}{\delta}\right)^{2}
$$

Let $v, \bar{v}$ be two solutions of (16) with initial value $v_{0}=u_{0} e^{z(\omega)}$ and $\bar{v}_{0}=$ $\bar{u}_{0} e^{z(\omega)}$.

If $v\left(t_{0}\right)-\bar{v}\left(t_{0}\right) \in \mathcal{K}_{\delta}$, then $v_{c}(t)-\bar{v}_{c}(t) \in \mathcal{K}_{\delta}$ for all $t \geq t_{0}$.

Moreover, if $v-\bar{v}$ is outside $\mathcal{K}_{\delta}$ at some time $t_{0}$ then

$$
\begin{aligned}
& \left\|v_{s}(t, \omega)-\bar{v}_{s}(t, \omega)\right\|^{2} \\
& \quad \leq\left\|u_{0}-\bar{u}_{0}\right\|^{2} \exp \left\{-\frac{1}{2} \lambda_{*} t+z(\omega)+2 \int_{0}^{t} z\left(\theta_{\tau} \omega\right) d \tau\right\}
\end{aligned}
$$

for all $t \in\left[0, t_{0}\right]$ 
In the proof we will see that the $4 \nu$ is not optimal in (25), but for simplicity of proof, we keep the $4 \nu$.

Proof. Define

$$
p=v_{c}-\bar{v}_{c} \text { and } q=v_{s}-\bar{v}_{s}
$$

then

$$
\begin{gathered}
\partial_{t} p=\nu p+z p+e^{-z} P_{c} B^{(R)}\left(v e^{z}\right)-e^{-z} P_{c} B^{(R)}\left(\bar{v} e^{z}\right) \\
\partial_{t} q=-L_{s} q+\nu q+z q+e^{-z} P_{s} B^{(R)}\left(v e^{z}\right)-e^{-z} P_{s} B^{(R)}\left(\bar{v} e^{z}\right) .
\end{gathered}
$$

By the property of operator $L$ and the Lipschitz property of $B^{(R)}$ we obtain for some positive constant $c_{1}$ depending on $\lambda_{*}$ and $\alpha$

$$
\frac{1}{2} \frac{d}{d t}\|p\|^{2} \geq \nu\|p\|^{2}+z\|p\|^{2}-L_{R}\|p\|^{2}-L_{R}\|p\|\|q\|
$$

and

$$
\frac{1}{2} \frac{d}{d t}\|q\|^{2} \leq-\|q\|_{1}^{2}+\nu\|q\|^{2}+z\|q\|^{2}+L_{R}\|q\|\|q\|_{\alpha}+L_{R}\|p\|\|q\|_{\alpha} .
$$

Thus

$$
\begin{gathered}
\frac{1}{2} \frac{d}{d t}\left(\|q\|^{2}-\delta^{2}\|p\|^{2}\right) \\
\leq \quad-\|q\|_{1}^{2}+\nu\|q\|^{2}+z\|q\|^{2}+L_{R}\|q\|\|q\|_{\alpha}+L_{R}\|p\|\|q\|_{\alpha} \\
\quad-\nu \delta^{2}\|p\|^{2}-z \delta^{2}\|p\|^{2}+\delta^{2} L_{R}\|p\|^{2}+\delta^{2} L_{R}\|p\|\|q\| .
\end{gathered}
$$

Now suppose that $v-\bar{v} \in \mathcal{K}_{\delta}$ (i.e. $\delta\|p\|=\|q\|$ ) for some $t$. Then

$$
\begin{aligned}
& \frac{1}{2} \frac{d}{d t}\left(\|q\|^{2}-\delta^{2}\|p\|^{2}\right) \\
& \quad \leq-\|q\|_{1}^{2}+L_{R}\|q\|\|q\|_{\alpha}+L_{R}\|p\|\|q\|_{\alpha}+\delta^{2} L_{R}\|p\|^{2}+\delta^{2} L_{R}\|p\|\|q\| \\
& \quad \leq-\|q\|_{1}^{2}+L_{R}(\|p\|+\|q\|)\|q\|_{1}+\delta^{2} L_{R}\|p\|^{2}+\delta^{2} L_{R}\|p\|\|q\| \\
& \quad \leq-\|q\|_{1}^{2}+L_{R}\left(1+\frac{1}{\delta}\right)\|q\|\|q\|_{1}+(1+\delta) L_{R}\|q\|^{2} \\
& \quad \leq-\frac{1}{2}\|q\|_{1}^{2}+\left(\frac{1}{2} L_{R}^{2}\left(1+\frac{1}{\delta}\right)^{2}+(1+\delta) L_{R}\right)\|q\|^{2}
\end{aligned}
$$

where we used Young inequality in the last step.

By (24) we obtain using Poincare inequality

$$
\frac{d}{d t}\left(\|q\|^{2}-\delta^{2}\|p\|^{2}\right) \leq-\frac{1}{2} \lambda_{*}\|q\|^{2}
$$


which yields the desired cone invariance.

For the second claim consider now that if $p+q$ is outside the cone at time $t_{0}$ ( i.e., $\left\|q\left(t_{0}\right)\right\|>\delta\left\|p\left(t_{0}\right)\right\|$ ). Then by the first result we have $\|q(t)\|>\delta\|p(t)\|$ for $t \in\left[0, t_{0}\right]$. Then by (28) we derive

$$
\begin{aligned}
\frac{1}{2} \frac{d}{d t}\|q\|^{2} & \leq-\|q\|_{1}^{2}+(\nu+z)\|q\|^{2}+L_{R}\|q\|\|q\|_{1}+L_{R}\|p\|\|q\|_{1} \\
& \leq-\|q\|_{1}^{2}+(\nu+z)\|q\|^{2}+L_{R}\left(1+\frac{1}{\delta}\right)\|q\|\|q\|_{1} \\
& \leq-\frac{1}{2}\|q\|_{1}^{2}+\left(\nu+z+\frac{1}{2} L_{R}^{2}\left(1+\frac{1}{\delta}\right)^{2}\right)\|q\|^{2}
\end{aligned}
$$

By (25)

$$
\frac{d}{d t}\|q(t, \omega)\|^{2} \leq\left(-\frac{1}{2} \lambda_{*}+2 z\left(\theta_{t} \omega\right)\right)\|q(t, \omega)\|^{2} .
$$

Then a comparison principle yields for almost all $\omega$

$$
\|q(t, \omega)\|^{2} \leq\|q(0, \omega)\|^{2} \exp \left\{-\frac{1}{2} \lambda_{*} t+2 \int_{0}^{t} z\left(\theta_{\tau} \omega\right) d \tau\right\} .
$$

Finally, $\|q(0, \omega)\|=\left\|v_{s}(0, \omega)-\bar{v}_{s}(0, \omega)\right\| \leq\left\|u_{0}-\bar{u}_{0}\right\| e^{z(\omega)}$ yields the result.

To finish the proof of Theorem 2, we also need the following lemma.

Lemma 2. For any given $T>0$, the following initial value problem

$$
\begin{aligned}
& \dot{v}_{c}=\nu v_{c}+z v_{c}+P_{c} e^{-z} B^{(R)}\left(\left(v_{c}+v_{s}\right) e^{z}\right), \quad v_{c}(T)=\xi \in H_{c} \\
& \dot{v}_{s}=\left(-L_{s}+\nu+z\right) v_{s}+P_{s} e^{-z} B^{(R)}\left(\left(v_{c}+v_{s}\right) e^{z}\right), \quad v_{s}(0)=h^{s}\left(v_{c}(0)\right)
\end{aligned}
$$

has a unique solution $\left(v_{c}(t, \omega), v_{s}(t, \omega)\right) \in C\left(0, T ; H_{c} \times H_{s}\right)$ which lies on the manifold $\mathcal{M}_{c u t}^{R}\left(\theta_{t} \omega\right)$ a.a..

Proof. This proof is same as Lemma 3.3 and Lemma 3.8 of [16] except that one should be careful of the nonlinearity is not Lipschitz on space $H$ in proving the contraction property of the solving operator. This step is same as the proof of contraction property of operator $\mathcal{T}$ defined in (18).

Now following the contradicting discussion in 24] we verify the following attracting property of the random invariant manifold.

Theorem 5. Assume (20), (24) and (25). For any solution $u(t, \omega)$ of cut-off system (15), there is one orbit $U(t, \omega)$ on $\mathcal{M}_{c u t}^{R}\left(\theta_{t} \omega\right)$ with $P_{c} U(t, \omega)$ solves the following equation

$$
\partial_{t} u_{c}=\nu u_{c}+P_{c} B^{(R)}\left(u_{c}+e^{z\left(\theta_{t} \omega\right)} h\left(\theta_{t} \omega, e^{-z\left(\theta_{t} \omega\right)} u_{c}\right)\right)+\sigma u_{c} \circ \dot{W}(t)
$$


such that

$$
\|u(t, \omega)-U(t, \omega)\| \leq D(t, \omega)\|u(0, \omega)-U(0, \omega)\| e^{-\lambda_{*} t}
$$

where $D(t, \omega)$ is a tempered increasing process defined by

$$
D(t, \omega)=e^{z\left(\theta_{t} \omega\right)+\int_{0}^{t} z\left(\theta_{\tau} \omega\right) d \tau} .
$$

Then Theorem 2 is a direct result of Theorem 5. Moreover we have the following result.

Corollary 1. There is a $\operatorname{LRIM} \mathcal{M}(\omega)$ for system (4) in a small ball $B(0, R)$ with $R$ and $\nu$ satisfy (20), (24), and (25). Moreover for $\left\|u_{0}\right\|<R$

$$
\operatorname{dist}\left(\varphi(t, \omega) u_{0}, \mathcal{M}(\omega)\right) \leq 2 R D(t, \omega) e^{-\lambda_{*} t}
$$

for all $t \in\left[0, \tau_{0}\right)$ where $\tau_{0}=\inf \left\{t>0: \varphi(t, \omega) u_{0} \notin B_{r}(0)\right\}$.

\section{Shape of the Invariant Manifold}

In this section we show that the LRIM $\mathcal{M}_{c u t}^{R}$, see (22), which denotes the random invariant manifold of equation (15) is locally quadratic. That is the Lipshitz-function $h$ (cf. Theorem 4) describing the manifold is quadratic near the fixed point 0 . Suppose $\sigma$ and $\nu$ are small and choose the cut-off $R$ also small. Consider first the LRIM of the transformed equation (16). We saw in the previous Section that it is given by

$$
\tilde{\mathcal{M}}_{c u t}^{R}(\omega)=\operatorname{Graph}(h)=\left\{(\xi, h(\omega, \xi)): \xi \in H_{c}\right\},
$$

where

$$
h(\omega, \xi)=P_{s} v^{*}(0, \omega, \xi)=v_{s}^{*}(0, \omega, \xi)
$$

with $v^{*}$ the unique fixed point of the contraction $\mathcal{T}$ in the space $C_{\eta}^{-}$(i.e., $v^{*}=\mathcal{T}\left(v^{*}\right)$, cf. Theorem (4). Thus for any $\xi \in H_{c}$ and $t \leq 0$

$$
\begin{aligned}
v^{*}(t)= & e^{\nu t+\int_{0}^{t} z(s) d s} \xi+\int_{0}^{t} e^{\nu(t-\tau)+\int_{\tau}^{t} z(r) d r} e^{-z(\tau)} B_{c}^{(R)}\left(e^{z(\tau)} v^{*}(\tau)\right) d \tau \\
& +\int_{-\infty}^{t} e^{\left(-L_{s}+\nu\right)(t-\tau)+\int_{\tau}^{t} z(r) d r} e^{-z(\tau)} B_{s}^{(R)}\left(e^{z(\tau)} v^{*}(\tau)\right) d \tau
\end{aligned}
$$

It was also established in the previous section, that the local invariant manifold for the original equation (44) is given by (cf. (23))

$$
\mathcal{M}^{R}(\omega)=\left\{\left(\xi, e^{z(\omega)} h\left(\omega, e^{-z(\omega)} \xi\right)\right): \xi \in H_{c}\right\} \cap B_{R}(0) .
$$

In order to describe the shape of the LRIM, we first establish two bounds on $v^{*}$ in $C_{\eta}^{-}$. 
Lemma 3. Under Assumptions $A_{1}-A_{3}$ suppose $\lambda_{*}>\nu+\eta>0$. Then there is a constant $C>0$ such that for all $\xi \in H_{c}$

$$
\left\|v^{*}\right\|_{C_{\eta}^{-}} \leq C\|\xi\| \quad \text { and } \quad\left\|v_{s}^{*}\right\|_{C_{\eta}^{-}} \leq C R\|\xi\| .
$$

Proof. For the first bound note that $\mathcal{T}(0)=e^{\nu t+\int_{0}^{t} z(s) d s} \xi$. Thus

$$
\|\mathcal{T}(0)\|_{C_{\eta}^{-}}=\sup _{t \leq 0} e^{(\nu+\eta) t}\|\xi\|=\|\xi\|
$$

Now as $\mathcal{T}$ is a contraction

$$
\left\|v^{*}\right\|_{C_{\eta}^{-}} \leq\left\|\mathcal{T}\left(v^{*}\right)-\mathcal{T}(0)\right\|_{C_{\eta}^{-}}+\|\mathcal{T}(0)\|_{C_{\eta}^{-}} \leq \operatorname{Lip}(\mathcal{T})\left\|v^{*}\right\|_{C_{\eta}^{-}}+\|\xi\| .
$$

We obtain the first claim with $C=1 /(1-\operatorname{Lip}(\mathcal{T})))$.

For the second claim we bound (30) by using the semigroup estimate from (19) together with the fact that

$$
\left\|B_{s}^{(R)}\left(v e^{z}\right)\right\|_{-\alpha} \leq C R\|v\| e^{z},
$$

for $v \in H$, which follows immediately from Definition 7 and Assumption $\mathrm{A}_{3}$. Thus

$$
\begin{aligned}
& \left\|v_{s}^{*}(t)\right\|=\left\|P_{s} v^{*}(t)\right\| \\
& =\left\|\int_{-\infty}^{t} e^{\left(-L_{s}+\nu\right)(t-\tau)+\int_{\tau}^{t} z(r) d r} e^{-z(\tau)} B_{s}^{(R)}\left(e^{z(\tau)} v^{*}(\tau)\right) d \tau\right\| \\
& \leq C \int_{-\infty}^{t}(t-\tau)^{-\alpha} e^{-(\lambda-\nu)(t-\tau)+\int_{\tau}^{t} z(r) d r} R\left\|v^{*}(\tau)\right\| d \tau \\
& \leq C R e^{\int_{0}^{t} z(r) d r} \int_{-\infty}^{t}(t-\tau)^{-\alpha} e^{-(\lambda-\nu)(t-\tau)} e^{-\eta \tau} d \tau\left\|v^{*}\right\|_{C_{\eta}^{-}} .
\end{aligned}
$$

Then using the first claim yields

$$
\left\|v_{s}^{*}\right\|_{C_{\eta}^{-}} \leq C R\|\xi\| \int_{-\infty}^{0}|\tau|^{-\alpha} e^{-(\lambda-\nu-\eta)|\tau|} d \tau
$$

Choosing $\lambda>\nu+\eta$ finishes the proof.

Now we prove the first reduction step, which removes the explicit dependence of $h$ on $v_{s}^{*}$. Define for $t \leq 0$ the cut-off $\chi_{R}^{*}(t)=\chi_{R}\left(v^{*}(t) e^{z(t)}\right)$ and the approximation

$$
g_{1}^{*}(t)=\int_{-\infty}^{t} e^{\left(-L_{s}+\nu\right)(t-\tau)+\int_{\tau}^{t} z(r) d r} \chi_{R}^{*}(\tau) B_{s}\left(v_{c}^{*}(\tau), v_{c}^{*}(\tau)\right) e^{z(\tau)} d \tau
$$


Lemma 4. Under Assumptions $A_{1}-A_{3}$ suppose $\lambda_{*}>\eta+\nu>0$, then

$$
\left\|v_{s}^{*}-g_{1}^{*}\right\|_{C_{\eta}^{-}} \leq C R^{2}\|\xi\| \quad \text { for all } \xi \in H_{c} .
$$

Proof. We use

$$
\begin{aligned}
e^{-z} B_{s}^{(R)}\left(e^{z} v^{*}\right) & =\chi_{R}^{*}(t) B_{s}\left(v^{*}, v^{*}\right) e^{z} \\
& =\chi_{R}^{*}(t)\left[B_{s}\left(v_{c}^{*}, v_{c}^{*} e^{z}\right)+2 B_{s}\left(v_{s}^{*}, v_{c}^{*} e^{z}\right)+B_{s}\left(v_{s}^{*}, v_{s}^{*} e^{z}\right)\right] .
\end{aligned}
$$

Now from

$$
\left\|\chi_{R}^{*} v_{c}^{*} e^{z}\right\| \leq R \quad \text { and } \quad\left\|\chi_{R}^{*} v_{s}^{*} e^{z}\right\| \leq R
$$

we obtain

$$
\left\|e^{-z} B_{s}^{(R)}\left(e^{z} v^{*}\right)-\chi_{R}^{*}(t) B_{s}\left(v_{c}^{*}, v_{c}^{*} e^{z}\right)\right\|_{-\alpha} \leq C R\left\|v_{s}^{*}\right\| .
$$

Analogously to (32) we derive with $\lambda \in\left(\eta+\nu, \lambda_{*}\right)$ that

$$
\begin{aligned}
\left\|v_{s}^{*}(t)-g_{1}^{*}(t)\right\| & \leq C \int_{-\infty}^{t}(t-\tau)^{-\alpha} e^{-(\lambda-\nu)(t-\tau)+\int_{\tau}^{t} z(r) d r} R\left\|v_{s}^{*}(\tau)\right\| d \tau \\
& \leq C \int_{-\infty}^{0}|\tau|^{-\alpha} e^{-(\lambda-\nu-\eta)|\tau|} d \tau \cdot e^{\int_{0}^{t} z(r) d r} e^{-\eta t} R\left\|v_{s}^{*}\right\|_{C_{\eta}^{-}}
\end{aligned}
$$

Thus using Lemma 3, the claim follows.

Recall that in order to bound $h$, we only need to consider $v_{s}^{*}(0)$. From (33)

$$
g_{1}^{*}(0)=\int_{-\infty}^{0} e^{L_{s} \tau} e^{-\nu \tau} e^{\int_{\tau}^{0} z(r) d r} \chi_{R}^{*}(\tau) B_{s}\left(v_{c}^{*}(\tau), v_{c}^{*}(\tau)\right) e^{z(\tau)} d \tau
$$

where

$$
v_{c}^{*}(\tau) e^{-\nu \tau} e^{\int_{\tau}^{0} z(r) d r}-\xi=\int_{0}^{\tau} e^{-\nu r+\int_{r}^{0} z(t) d t} e^{z(r)} \chi_{R}^{*}(r) B_{c}\left(v^{*}(r), v^{*}(r)\right) d r .
$$

Using $B_{c}\left(v_{c}^{*}, v_{c}^{*}\right)=0$ and thus

$$
e^{z} \chi_{R}^{*} B_{c}\left(v^{*}, v^{*}\right)=2 B_{c}\left(v_{s}^{*}, e^{z} \chi_{R}^{*} v_{c}^{*}\right)+B_{c}\left(v_{s}^{*}, e^{z} \chi_{R}^{*} v_{s}^{*}\right)
$$

we obtain for $\tau \leq 0$

$$
\begin{aligned}
& \left\|v_{c}^{*}(\tau) e^{-\nu \tau} e^{\int_{\tau}^{0} z(r) d r}-\xi\right\| \\
& \quad \leq \int_{\tau}^{0} e^{-\nu r+\int_{r}^{0} z(t) d t} R\left\|v_{s}^{*}(r)\right\| d r \\
& \quad \leq R \int_{\tau}^{0} e^{-(\nu+\eta) r} d r\left\|v_{s}^{*}\right\|_{C_{\eta}^{-}} \leq C R^{2}\|\xi\| e^{-(\nu+\eta) \tau} .
\end{aligned}
$$


Hence,

$$
\left\|v_{c}^{*}(\tau)-\xi e^{\nu \tau} e^{\int_{0}^{\tau} z(r) d r}\right\| \leq C R^{2}\|\xi\| e^{-\eta \tau} e^{\int_{0}^{\tau} z(r) d r}
$$

Thus for

$$
g_{2}^{*}:=\int_{-\infty}^{0} e^{L_{s} \tau} \chi_{R}^{*}(\tau) B_{s}\left(\xi, v_{c}^{*}(\tau)\right) e^{z(\tau)} d \tau
$$

we obtain using (34) and estimates analogous to (32)

$$
\begin{aligned}
\left\|g_{1}^{*}(0)-g_{2}^{*}\right\| & \leq C \int_{-\infty}^{0}|\tau|^{-\alpha} e^{-\lambda|\tau|} R \cdot\left\|e^{-\nu \tau} e^{\int_{\tau}^{0} z(r) d r} v_{c}^{*}(\tau)-\xi\right\| d \tau \\
& \leq C R^{3}\|\xi\| \int_{-\infty}^{0}|\tau|^{-\alpha} e^{-\lambda|\tau|} e^{-(\nu+\eta) \tau} d \tau
\end{aligned}
$$

Thus together with Lemma 4 we have

Theorem 6. Under Assumptions $A_{1}-A_{3}$ suppose $R \leq 1$, and $\lambda_{*}>\nu+\eta>0$. Then

$$
\left\|h(\omega, \xi)-g_{2}^{*}(\omega, \xi)\right\| \leq C R^{2}\|\xi\| \quad \text { for all } \xi \in H_{c} .
$$

This theorem is how far we can get without $\omega$-dependent deterministic bounds. In the following we will rely on the following lemma (cf. (9) ).

Lemma 5. There is a random variable $K(\omega)$ such that $K(\omega)-1$ has a standard exponential distribution and for all $t \leq 0$

$$
\int_{0}^{t} z(r) d r+z(t)=z(0)+\sigma \omega(t) \leq \sigma(K(\omega)+|t|) .
$$

Proof. Define

$$
\tilde{K}(\omega)=\sup _{s \leq 0}\{\omega(s)+s\} .
$$

For example from [26] we know that $2 \tilde{K}$ has a standard exponential distribution. Obviously,

$$
\sigma \omega(t) \leq \sigma(\tilde{K}(\omega)+|t|) \text { for all } t \leq 0 .
$$

Moreover, by definition

$$
\begin{aligned}
z(0) & =\sigma \int_{-\infty}^{0} e^{s} \omega(s) d s \\
& \leq \sigma \int_{-\infty}^{0} e^{s} d s \tilde{K}(\omega)-\sigma \int_{-\infty}^{0} s e^{s} d s=\sigma(\tilde{K}(\omega)+1)
\end{aligned}
$$


Remark 3. For $s<0$ we have

$$
|\omega(s)| \leq \max \{\omega(s),-\omega(s)\} \leq K^{ \pm}(\omega)+|s|,
$$

where

$$
K^{ \pm}(\omega)=K(\omega)+K(-\omega)
$$

and $K(-\omega)$ has the same law as $K(\omega)$. Furthermore, for $|z(0)|$ a similar estimate is true.

Define

$$
g_{3}^{*}:=\int_{-\infty}^{0} e^{L_{s} \tau} \chi_{R}^{*}(\tau) e^{z(\tau)} e^{\nu \tau} e^{\int_{0}^{\tau} z(r) d r} d \tau B_{s}(\xi, \xi) .
$$

Using (34) yields

$$
\begin{aligned}
\left\|g_{2}^{*}-g_{3}^{*}\right\| & \leq C \int_{-\infty}^{0}|\tau|^{-\alpha} e^{-\lambda|\tau|} e^{z(\tau)}\|\xi\| \cdot\left\|v_{c}^{*}(\tau)-\xi e^{\nu \tau} e^{\int_{0}^{\tau} z(r) d r}\right\| d \tau \\
& \leq C R^{2}\|\xi\|^{2} \int_{-\infty}^{0}|\tau|^{-\alpha} e^{-\lambda|\tau|} e^{\int_{0}^{\tau} z(r) d r} e^{z(\tau)} e^{-\eta \tau} d \tau
\end{aligned}
$$

Then by Lemma 5 we obtain for $\sigma<\frac{1}{2}(\lambda-\eta)$

$$
\begin{aligned}
\left\|g_{2}^{*}-g_{3}^{*}\right\| & \leq C R^{2}\|\xi\|^{2} \int_{-\infty}^{0}|\tau|^{-\alpha} e^{(\eta-\lambda+2 \sigma)|\tau|} e^{2 \sigma K(\omega)} d \tau \\
& \leq C R^{2}\|\xi\|^{2} e^{2 \sigma K(\omega)} .
\end{aligned}
$$

Now we eliminate the cut-off. Suppose $\|\xi\| e^{z(0)} \leq R$, then we can use the smoothness of $\chi_{R}$ (mean value theorem) to obtain

$$
\begin{aligned}
\left|\chi_{R}^{*}(\tau)-1\right| & =\left|\chi_{R}\left(v^{*}(\tau) e^{z(\tau)}\right)-\chi_{R}\left(\xi e^{z(0)}\right)\right| \\
& \leq \frac{C}{R}\left\|v_{s}^{*}(\tau) e^{z(\tau)}\right\|+\frac{C}{R}\left\|v_{c}^{*}(\tau) e^{z(\tau)}-\xi e^{z(0)}\right\| \\
& \leq C(1+R)\|\xi\| e^{-\eta \tau+z(\tau)+\int_{0}^{\tau} z(r) d r}+\frac{C}{R}\|\xi\| \cdot\left|e^{z(0)}-e^{\nu \tau+\int_{0}^{\tau} z(r) d r}\right|
\end{aligned}
$$

where we used Lemma 3 and (34).

Now from Lemma 5, and the fact that $z(t)+\int_{0}^{t} z(r) d r=z(0)+\sigma \omega(t)$ we derive

$$
\left|\chi_{R}^{*}(\tau)-1\right| \leq C(1+R)\|\xi\| e^{-\eta \tau+\sigma|\tau|+\sigma K(\omega)}+C\left|1-e^{\nu \tau+\sigma \omega(\tau)}\right| e^{z(0)} .
$$

Thus

$$
\left|\chi_{R}^{*}(\tau)-1\right| \leq C\left(\|\xi\|(1+R)+\sigma K_{2}(\omega)\right) e^{-\eta \tau} e^{\sigma|\tau|+\sigma K(\omega)}
$$


where we define

$$
K_{2}(\omega):=\sup _{\tau \leq 0}\left|\frac{1-e^{\nu \tau+\sigma \omega(\tau)}}{\sigma e^{\eta|\tau|}}\right|
$$

For this random constant $K_{2}$ we obtain the following relation to $K(\omega)$. Note that this result is by far not optimal, but it is rather simple to derive.

Lemma 6. Suppose $|\nu|+|\sigma|<\frac{\eta}{2}$ and $|\nu| \leq|\sigma|$, then there is a constant depending only on $\eta$ such that

$$
K_{2}(\omega) \leq C e^{|\sigma| K^{ \pm}(\omega)}\left(1+K^{ \pm}(\omega)\right) .
$$

Proof. We use the rather crude estimate $\left|1-e^{x}\right| \leq|x| e^{|x|}$ for all $x \in \mathbb{R}$. Recall Remark 3 to obtain

$$
\begin{aligned}
K_{2}(\omega) & \leq \sup _{\tau \leq 0} \frac{|\nu||\tau|+|\sigma||\omega|}{|\sigma|} e^{|\nu||\tau|+|\sigma \omega(\tau)|-\eta|\tau|} \\
& \leq \sup _{\tau \leq 0} \frac{(|\nu|+|\sigma|)|\tau|+|\sigma|}{|\sigma| K^{ \pm}(\omega)} e^{|\sigma| K^{ \pm}(\omega)} e^{|\tau|(|\nu|+|\sigma|-\eta)} \\
& \leq 2 e^{|\sigma| K^{ \pm}(\omega)} \sup _{\tau \leq 0}\left(|\tau|+K^{ \pm}(\omega)\right) e^{-\frac{1}{2}|\tau| \eta} .
\end{aligned}
$$

Now we proceed to bound $g_{3}^{*}$

$$
\begin{aligned}
& \left\|g_{3}^{*}-\int_{-\infty}^{0} e^{L_{s} \tau} e^{z(\tau)} e^{\nu \tau} e^{\int_{0}^{\tau} z(r) d r} d \tau B_{s}(\xi, \xi)\right\| \\
& \quad \leq C \int_{-\infty}^{0}|\tau|^{-\alpha} e^{(-\lambda+2 \sigma)|\tau|} e^{\sigma K(\omega)}\left|1-\chi_{R}^{*}(\tau)\right| d \tau\|\xi\|^{2} \\
& \stackrel{400}{\leq} C \int_{-\infty}^{0}|\tau|^{-\alpha} e^{(-\lambda+\eta+2 \sigma)|\tau|} e^{2 \sigma K(\omega)} d \tau\left(\|\xi\|(1+R)+\sigma K_{2}(\omega)\right)\|\xi\|^{2} \\
& \leq C e^{2 \sigma K(\omega)}\left(\|\xi\|+\sigma K_{2}(\omega)\right)\|\xi\|^{2}
\end{aligned}
$$

for $R \leq 1$ and $\eta-\lambda+2 \sigma<0$.

For the final step note that

$$
\int_{-\infty}^{0} e^{L_{s} \tau} e^{z(\tau)} e^{\nu \tau} e^{\int_{0}^{\tau} z(r) d r} d \tau=e^{z(0)} \int_{-\infty}^{0} e^{L_{s} \tau} e^{\nu \tau} e^{\sigma \omega(\tau)} d \tau
$$


We finally bound

$$
\begin{aligned}
& \left\|\int_{-\infty}^{0} e^{L_{s} \tau} e^{\nu \tau} e^{\sigma \omega(\tau)} d \tau B_{s}(\xi, \xi)-L_{s}^{-1} B_{s}(\xi, \xi)\right\| \\
& =\left\|\int_{-\infty}^{0} e^{L_{s} \tau}\left(1-e^{\nu \tau} e^{\sigma \omega(\tau)}\right) d \tau B_{s}(\xi, \xi)\right\| \\
& \leq C \int_{-\infty}^{0}|\tau|^{-\alpha} e^{(-\lambda+\eta)|\tau|} \sigma K_{2}(\omega) d \tau\|\xi\|^{2} \\
& \leq C \sigma K_{2}(\omega)\|\xi\|^{2}
\end{aligned}
$$

for $\lambda>\eta$.

Combining (39), (42), and (43), we proved the following theorem:

Theorem 7. Let Assumptions $A_{1}-A_{3}$ be true. Suppose $\lambda_{*}>\eta+2 \sigma$, $\lambda_{*}>\nu+\eta, \sigma>0$, and $\|\xi\| e^{z(0)} \leq R \leq 1$, and let $h$ be the fixed point given by Theorem 4. Then

$$
\left\|h(\omega, \xi)-e^{z(0)} L_{s}^{-1} B_{s}(\xi, \xi)\right\| \leq C\left(e^{2 \sigma K(\omega)}+1\right) \cdot\left(\|\xi\|+R^{2}+\sigma K_{2}(\omega)\right) \cdot\|\xi\|^{2},
$$

where $K$ and $K_{2}$ are defined in (36) and (41).

This is the final main Theorem of this section. The main Theorem 3 is now a simple corollary.

Proof of Theorem [3. Define

$$
\Omega_{K}=\left\{\omega \in \Omega_{0}: K^{ \pm}(\omega)>1 / \sqrt{\sigma}\right\} .
$$

By Lemma 5 this set has probability less that $C \exp \{-1 / \sqrt{\sigma}\}$. Moreover, on the complement $\Omega_{K}^{c}$ we have

$$
K \leq \frac{1}{\sqrt{\sigma}} \quad \text { and } \quad K_{2} \leq \frac{1}{\sqrt{\sigma}}
$$

and (13) follows from Theorem 7, as similar to Lemma 6 we also have on $\Omega_{K}^{c}$

$$
e^{z(0)}\|\xi\| \leq e^{C \sqrt{\sigma}}\|\xi\| \leq R .
$$

\section{Acknowledgments}

Supported by Bosch-foundation Nr. 32.5.8003.0010.0 ("Angewandte Mathematik") and NSFC No. 10701072. Part of the work was done during a stay of W. Wang at the University of Augsburg. 


\section{References}

[1] L. Arnold, Random Dynamical Systems, Springer-Verlag, New York/Berlin, 1998.

[2] D. Blömker, Amplitude equations for locally cubic non-autonomous nonlinearities, SIAM J. on Appl. Dyn. Sys. 2(2),(2003), 464-486.

[3] D. Blömker \& M. Hairer, Multiscale expansion of invariant measures for SPDEs, Commun. in Math. Phys. 251(3),(2004), 515-555.

[4] D. Blömker, Amplitude Equations for Stochastic Partial Differential Equations, Vol. 3 of Interdisciplinary Mathematical Sciences, World Scientific Publishing, 2007.

[5] D. Blömker, M. Hairer, \& G. Pavliotis. Multiscale analysis for SPDEs with quadratic nonlinearities. Nonlinearity, 20, (2007) 1721-1744.

[6] D. Blömker, M. Hairer, \& G. Pavliotis. Stochastic Swift-Hohenberg equation near a change of stability. pp. 27-37 in Proceedings of Equadiff 11, Proceedings of minisymposia and contributed talks, July 2529, 2005, Bratislava, (Ed. M. Fila, A. Handlovicova, K. Mikula, M. Medved, P. Quittner \& D. Sevcovic), 2007.

[7] D. Blömker, F. Flandoli \& M. Romito, Markovianity and ergodicity for a surface growth PDE, to appear in Annals of Probability

[8] T. Caraballo, J. A. Langa \& J.C.Robinson, Stability and random attractors for a reaction-diffusion equation with multiplicative noise, Disc. and Cont. Dyn. Sys. 6 (2000), 875-892.

[9] T. Caraballo, J. A. Langa \& J.C.Robinson, A stochastic pitchfork bifurcation in a reaction-diffusion equation, Proc. Roy. Soc. London A 457 (2001), 2041-2061.

[10] I. D. Chueshov, J. Duan \& B. Schmalfuß, Determining functionals for random partial differential equations, NoDEA 10 (2003), 431-454.

[11] I. D.Chueshov, Approximate inertial manifolds of exponential order for semilinear parabolic equations subjected to additive white noise, $J$. Dyn. and Diff. Equ. 7(4)(1995), 549-566.

[12] H. Crauel \& F. Flandoli, Attractor for random dynamical systems, Prob. Theory and Relat. Fields. 100 (1994), 365-393. 
[13] H. Crauel, A. Debussche \& F. Flandoli, Random attractors, J. Dyn. Diff. Equ. 9(2) (1997), 307-341.

[14] G. Da Prato \& J. Zabczyk, Stochastic Equations in Infinite Dimensions, Cambridge University Press, 1992.

[15] J. Duan, X. Li \& X. Sun. An impact of noise on invariant manifolds in dynamical systems. In Preparation.

[16] J. Duan, K. Lu \& B. Schmalfuß. Invariant manifolds for stochastic partial differential equations, Ann. Prob., 31(4) (2003), 2109-2135.

[17] J. Duan, K. Lu \& B. Schmalfuß. Smooth stable and unstable manifolds for stochastic partial differential equations, J. Dyn. and Diff. Equ., 16 (4) (2004), 949-972.

[18] J. Garcia-Ojalvo \& J. M. Sancho, Noise in Spatially Extended Systems. Springer-Verlag, 1999.

[19] Z. Huang \& J. Yan, Introduction to Infinite Dimensional Stochastic Analysis. Science Press/Kluwer Academic Pub., Beijing/New York, 1997.

[20] K. Lu \& B. Schmalfuß, Invariant manifolds for stochastic wave equations, J. Diff. Equa. 236(2) (2007), 460-492.

[21] B. L. Rozovskii, Stochastic Evolution Equations. Kluwer Academic Publishers, Boston, 1990.

[22] B. Schmalfuß, Backward cocycles and attractors of stochastic differential equations, In International Seminar on Applied MathematicsNonlinear Dynamics: Attractor Approximation and Global Behaviour, V. Reitmann, T. Riedrich \& N. Koksch (Eds.), 1992, 185-192.

[23] R. Temam, Infinite-dimensional dynamical systems in mechanics and physics. Springer-Verlag, New York, second edition, 1997.

[24] W. Wang \& J. Duan, A dynamical approximation for stochastic partial differential equations, J. Math. Phys. 48, (2007), 102701-14.

[25] E. Waymire \& J. Duan (Eds.), Probability and Partial Differential Equations in Modern Applied Mathematics. IMA Volume 140, SpringerVerlag, New York, 2005. 
[26] M. Yor, Some remarks about the joint law of Brownian motion and its supremum. Azema, J. (ed.) et al., Seminaire de probabilites XXXI. Berlin: Springer. Lect. Notes Math. 1655, (1997), 306-314. 